\title{
Proyecto integrador de saberes, evidencia del resultado de aprendizaje
}

\section{Project integrating knowledge, evidence of learning outcome}

Víctor Hugo Moscoso Zamora

Erika Sonia Quiñonez Alvarado

Universidad de Guayaquil, Ecuador

Autor para correspondencia: victor.moscosoz@ug.edu.ec, erika.quinoneza@ug.edu.ec

Fecha de recepción: 12 de Octubre 2017 - Fecha de aceptación: 15 de Febrero de 2018

Resumen: Este trabajo describe la aplicación de didácticas que generarán una evidencia del logro y eficacia del aprendizaje en la educación superior, puesto que en Ecuador las universidades en busca de la excelencia académica están aplicando estrategias metodológicas progresivas en cada nivel de organización curricular, desde la etapa de admisión en los cursos de nivelación se establece vincular conocimientos y proponer un proyecto, esta tarea se encuentra normada por el Reglamento de Régimen Académico vigente (RRA), que establece el desarrollo del Proyecto integrador de Saberes (PIS) en cada uno de los semestres de toda carrera universitaria del país, lo que obliga a las Instituciones de Educación Superior a introducir y estructurar nuevos métodos de enseñanza que no solo combinen los conocimientos sino que además sirvan de evidencia del proceso de enseñanza. Para la elaboración de este estudió se aplicó una tipología descriptiva y el método analítico-sintético, se utilizaron fuentes documentales impresas y digitales. Se concluyó que al estructurar y orientar de manera correcta el proyecto integrador de saberes, a través de la interacción investigación-acción, permite al estudiante adquirir competencias de la profesión, que evidencian el resultado del aprendizaje.

Palabras Claves: excelencia académica; proyecto integrador; estrategias metodológicas; evidencia; resultado del aprendizaje

Abstract: This paper describes the application of didactics that will generate evidence of the achievement and effectiveness of learning in higher education, since in Ecuador the universities in search of academic excellence are applying progressive methodological strategies in each level of curricular organization, from the stage of Admission in the leveling courses, it is established to link knowledge and propose a project, this task is regulated by the Regulation of Academic Regime in force (RRA), which establishes the development of the Integrative Knowledge Project (PIS) in each of the semesters of Every university career in the country, which obliges institutions of higher education to introduce and structure new teaching methods that not only combine knowledge but also serve as evidence of the teaching process. For the elaboration of this study a descriptive typology was applied and the analytical-synthetic method used printed and digital documentary sources. It was concluded that by structuring and correctly orienting the integrative project of knowledge, through interaction research-action, allows the student to acquire competencies of the profession, which evidence the result of learning.

Key Words: academic excellence; integrative project; methodological strategies; evidence; learning outcome 


\section{Introducción}

Este documento tiene como objetivo principal; el dar a conocer que, el proyecto integrador de saberes aplicado como estrategia metodológica y de investigación-acción, incluido en los planes curriculares de la instituciones de educación superior, estructura un producto académico que evidencia el logro de los aprendizajes en cada nivel de la unidad de la organización curricular, fortaleciendo el desarrollo de las competencias académicas que articulen con el perfil de egreso del futuro profesional, además, incentiva al estudiante a resolver problemas de la profesión, mediante el trabajo en equipo.

Para la transmisión de conocimientos en un aula de clases, se utilizan una diversidad de actividades académicas aplicando la estrategia y metodología acorde con la finalidad de generar un resultado del aprendizaje eficaz, lo que se evidencia mediante un producto académico. La educación superior en el Ecuador, se encuentra en la búsqueda de un mejor nivel académico, especialmente en el proceso enseñanza-aprendizaje, por lo que se propone que, se debe generar un constructo en cada nivel de las carreras, que culmine con un resultado del aprendizaje en el mismo, el que a su vez aporte al perfil de egreso del futuro profesional.

En cuanto al perfil de egreso del futuro profesional, se establece deberá ser elaborado considerando las necesidades del contexto laboral, en este caso los objetivos del Plan Nacional del Buen vivir y los problemas y planes de las 9 zonas en que se encuentra dividido Ecuador, ya que cada una de ellas tienen propósitos de desarrollo distintos (SENPLADES, Secretaría Nacional de Planificación y Desarrollo, 2013).

Afianzar el conocimiento en el proceso enseñanza-aprendizaje mediante el PIS, un producto académico que integra los saberes en cada nivel de formación, es decir, un proyecto que sirva como indicador y evidencia de que el proceso logró su objetivo en la escala y nivel de formación correspondiente (Coordinación de formación Facultad de Ciencias Admiistrativas Universidad de Guayaquil, 2016). En este contexto, teniendo al estudiante como protagonista y al docente como facilitador de un proceso de formación integral (Larrea \& Montalván, 2016).

De acuerdo a Cruz y Matos (2017), es importante resaltar el reto para los docentes que faciliten la Cátedra Integradora en el proceso de gestión académico- investigativa, pues como condición indispensable, es necesario poseer una profunda formación en las esencialidades y actual desarrollo de la diversidad de ciencias que sustentan la profesión, así como una sentida formación didáctica que permita su integración funcional desde el salón de clases, en función de la diversidad de marcos teóricos y propuestas de investigación a construir. El docente, entonces, debe ser capaz de lograr un equilibrio entre lo individual y lo grupal, entre su propia formación previa y los desafíos de esta nueva propuesta, para poder satisfacer de manera dinámica e integradora las necesidades de los estudiantes de aprender a hacer una investigación educativa.

Para esta investigación se hace una revisión literaria para sustentar las bases del desarrollo del PIS y su papel trascendental en la educación superior; se establece el tipo de investigación utilizado para este artículo; se hacen conclusiones y recomendaciones basadas en los constructos teóricos revisados para llegar a la comprensión de lo plasmado en esta obra; se incluye un apéndice que contiene material que complementa el tema abordado en esta investigación. 


\section{Marco referencial teórico}

En el 2008 la Asamblea Nacional Constituyente expide el Mandato Constituyente No.14, que establece la obligación de elaborar un informe técnico sobre el nivel de desempeño institucional de los establecimientos de educación superior, a fin de garantizar su calidad, propiciando su depuración y mejoramiento, es así que en el 2010 el Consejo Nacional de Evaluación y Acreditación de la Educación Superior del Ecuador (CONEA) entrega la evaluación final donde se analiza la situación de la educación superior que revela el desarrollo de las Instituciones de Educación Superior (IES) del país, este estudio abarcaba las normas, prácticas inclusivas y pertinencia de la enseñanza, el resultado fue desalentador lo que requirió una reflexión y acción correctiva del gobierno a crear un proceso que revierta estos indicadores. Es así que se comienzan a redactar normativas que incluyen leyes con carácter de orgánicas y reglamentos en búsqueda de la excelencia académica de las universidades y escuelas politécnicas.

Pero que se considera excelencia académica, este término e relaciona con la calidad, de acuerdo al artículo 93 de Ley Orgánica de Educación Superior determina que el principio de calidad es la búsqueda constante y sistemática de la excelencia, la pertinencia, producción óptima, transmisión del conocimiento y desarrollo del pensamiento mediante (...) el mejoramiento permanente (Consejo Nacional de Evaluación y Acreditación de la Educación Superior del Ecuador, CONEA, 2009).

Según el Consejo de Educación Superior (CES) la calidad y el logro de la excelencia en la Universidad Ecuatoriana, se evidencian en indicadores propuestos por los organismos de control de la educación superior de nuestro país, y se consideran de mucha relevancia especialmente; el indicador de desarrollo de contextos pedagógicos y curriculares interactivos, creativos y de construcción innovadora del conocimiento.

Desde el indicador docente debe emanar la transmisión del conocimiento, y la responsabilidad de la formación del perfil del futuro profesional, es decir, generar un resultado del aprendizaje preestablecido y que se evidencie en un producto académico esperado que articule con las necesidades del contexto laboral.

El constructivismo según Jean Piaget, como corriente pedagógica, se ha mantenido en la Educación Superior, a pesar de algunos de sus detractores (Gaete 2013, Anguita, F. 2001 Chadwick, C. 2005), pues, didácticamente este proceso de enseñanza dota al estudiante de las herramientas necesarias para la construcción del conocimiento y la interacción con el medio (investigación acción), lo que le permitirá resolver problemas de la profesión.

Además, del desarrollo intelectual, que es la base fundamental para su aplicación en el contexto, experiencias que irán acumulándose y mediante la epistemología generará nuevas y mejores estructuras que resuelvan situaciones problemicas, incluso más complejas.

Según la (Secretaría de Educación Superior, Ciencia, Tecnología e Innovación, SENECYT, 2015) el Proyecto Integrador de Saberes es "el eje de producción de aprendizajes, 
que expresa los avances y logros educativos de los estudiantes en cada una de las unidades de análisis del proceso de nivelación”.

Con base a lo anteriormente expuesto, mediante la aplicación del modelo constructivista se elaboran los proyectos integradores de saberes, los que se desarrollan en nuestro país desde los cursos de nivelación y admisión (SNNA), y se establece como actividad de aprendizaje colaborativo en el Reglamento de Régimen Académico vigente (Art, 15 inc. b), y el grado de complejidad deberá aplicarse acorde al nivel de formación.

La Secretaria Nacional de Educación Superior, Ciencia, Tecnología e Innovación del Ecuador (SENECYT) describe al proyecto de integración de saberes como el eje de producción de aprendizajes, que expresa los avances y logros educativos de los estudiantes en las diferentes unidades de análisis, y por su carácter teórico-práctico, posibilita el desarrollo de habilidades en contexto de aplicación de conocimientos.

El Sistema Nacional de Nivelación y Admisión (SNNA) lleva a cabo los Proyectos Integradores de Saberes (PIS), el cual tiene como objetivo potencializar el desempeño de los estudiantes en el ciclo de pregrado, considerando la importancia de este proceso para la investigación científica en la etapa universitaria (Secretaría de Educación Superior, Ciencia, Tecnología e Innovación, SENECYT, 2015).

El proyecto integrador de saberes de acuerdo a Blank, (1997); Dickinson, (1998); Harwell, (1997) citados por Galeana (2017) es un modelo de enseñanza - aprendizaje que permite al alumnado ser el protagonista de su educación, vinculando la teoría y la práctica. Su realización involucra planeación y evaluación de acuerdo al entorno social, y su ejecución trasciende el salón de clases. Ayuda entonces a la medición del conocimiento asimilado por el educando.

Según (Gento, 1998) en su artículo "Marco referencial para la evaluación de un proyecto educativo, considera a la evaluación un componente ineludible de todo proyecto educativo que aspira a ser válido y eficaz, con el propósito de mejorar los resultados, optimizar el proceso de ejecución y, si fuera preciso reconsiderar los objetivos propuestos".

\section{Metodología}

Este artículo es de nivel descriptivo, puesto que se caracterizan los criterios que rigen el Proyecto Integrador de Saberes, se exploran las causas y su inferencia en la construcción de conocimientos, para explicarlos respecto al proceso de acreditación de la enseñanza y planes curriculares como muestra del saber adquirido, (Sampieri, Collado, \& Lucio, 2014), a fin de establecer sus implicaciones en la gestión académica (Arías, 2012).

El enfoque cualitativo, tiene como finalidad comprender, interpretar y reflexionar acerca de los resultados del aprendizaje usando el PIS como parte de la gestión formativa del estudiante (Arías, 2012). El diseño de este estudio es de carácter documental y bibliográfico, se realiza una revisión de la literatura y análisis de datos secundarios de fuentes impresas y electrónicas, como las normativas de educación superior vigentes en el Ecuador (R.R.A) y de investigadores con 
obras de complejidad sistémica que fueron examinadas y estudiadas con el fin de descifrar el sentido de las mismas e identificar vínculos e implicaciones (Arías, 2012). En cuanto al método aplicado en el desarrollo de este documento; fue el analítico-sintético, consiste en separar, analizar e indagar en el objeto de estudio, para luego integrar las partes de manera holística (Sampieri, Collado, \& Lucio, 2014).

\section{Estructura curricular}

La estructura curricular "es el conjunto de objetivos previamente establecidos y debidamente organizados" (Organización de las Naciones Unidas para la Agricultura y la Alimentación, FAO, 2011). Es relevante considerar, que todo inicia desde la Estructura Curricular de la carrera (Ver figura A1), pues es donde se establecen los conocimientos disciplinares, interdisciplinares, transdisciplinares, profesionales, investigativos, de saberes integrales y de comunicación, necesarios para desarrollar el perfil profesional y académico del estudiante, articulando los conocimientos de modo progresivo e integrador, a lo largo de la carrera o programa. La estructura curricular evidenciará la consistencia, coherencia y correspondencia interna entre: el perfil de ingreso, las relaciones entre los conocimientos y saberes del conjunto de las asignaturas, cursos o sus equivalentes y el perfil de egreso; aportando al desarrollo y fortalecimiento de las capacidades integrales de los futuros profesionales según el artículo 20, del R.R.A. (Consejo de Educación Superior, 2013).

El primer reto fue; el de acoplar la oferta académica actual a la nueva estructura curricular, ya que, los ejes transversales, como las Unidades de Organización Curricular (U.O.C) y los Campos de Formación (C.F) establecidos en el Reglamento de Régimen Académico, tienen una diferencia muy marcada y me atrevería a decir que hasta superior, comparada con la estructura anterior.

Esta nueva experiencia sirvió de plataforma a la Academia Ecuatoriana, para generar nuevas y mejores propuestas, que en cada nivel de formación (semestre), se debe iniciar con un núcleo problémicos para lo cual se integran las asignaturas correspondientes al mismo y se culmina con un resultado del aprendizaje, el cual, debe aportar a la construcción del perfil de Egreso, un logro que se dio gracias a la colaboración y el aporte de los docentes gestores pedagógicos, curriculares y de ambientes de aprendizaje, sin embargo, sería relevante también dejar evidenciado el resultado del aprendizaje.

\section{Evidencia del resultado del aprendizaje}

El Artículo 15, del Reglamento de Régimen Académico vigente, con respecto a las actividades de aprendizaje, en el inciso b, establece que entre las actividades de aprendizaje colaborativo se encuentra el Proyecto Integrador de Saberes, lo que implica su elaboración y desarrollo en el proceso educativo a lo largo de toda la formación profesional.

Para evidenciar un proceso de enseñanza-aprendizaje, cuya nueva estructura curricular se redirecciona, a mejorar la calidad de la Educación Superior desde la complejidad sistémica, se requiere de un carácter analítico-sintético, para la obtención de las metas de modo progresivo e integrador, dado que, el grado de complejidad de cada proyecto integrador de saberes deberá 
implementarse progresivamente acorde al nivel de formación del futuro profesional, el mismo que estructurado en forma correcta se convertiría en el producto académico esperado.

Para la consecución de un Proyecto Integrador de Saberes, que aporte al perfil de egreso y principalmente evidencie el logro de los objetivos del aprendizaje en cada nivel (semestre) de formación, se deberá constituir principalmente considerando; una catedra integradora que nazca de la praxis pre profesional (C.F), la misma que coordinará con una asignatura de fundamentación teórica y una asignatura de Investigación, estructurando así una trilogía perfecta de acuerdo a la Dra. Elizabeth Larrea.

Se necesita planificar la temática a desarrollar en cada semestre, cuyo nivel de complejidad deberá implementarse en forma secuencial y progresiva, articulando al perfil de egreso, cada asignatura correspondiente se integrará aportando a la construcción del proyecto, logrando así una interdependencia entre ellas, bajo la supervisión de cada uno de los docentes que imparten dichas cátedras, los cuales formarán grupos de trabajo, que se aconsejan no sean mayores a 5 estudiantes, a los que se le deberán asignar las tareas parciales que integradas evidenciarán el resultado del aprendizaje.

La coordinación por parte del docente que imparte la catedra integradora, es fundamental, puesto que conduce las etapas del proyecto según el plan de las orientaciones metodológicas en cada etapa modular de la trayectoria curricular sin dejar de lado la asistencia e integración de los catedráticos de las asignaturas colaborativas que aportan con bases teóricas las propuestas dependiendo el nivel del curso.

\section{Conclusiones}

La Universidad Ecuatoriana, está dando grandes pasos hacia la excelencia académica, mediante estrategias metodológicas aplicadas a la nueva estructura curricular vigente, por lo que se torna de mucha relevancia, dejar constancia no solo del logro del resultado del aprendizaje, sino también, de la eficacia del mismo. Por lo tanto, el estructurar y orientar de manera correcta el Proyecto integrador de Saberes donde interactúan investigación-acción, permitirá al estudiante aprender a resolver en forma progresiva problemas de la profesión, y así mismo evidenciar el desarrollo de las habilidades acordes al perfil de egreso. Por tanto, el PIS sirve de medición del resultado del proceso académico, convirtiéndose en un verdadero acierto.

Cabría, por tanto, incluir normas y criterios de validación para la medición de los resultados a través de indicadores que manifiesten la eficacia del PIS en relación: a los objetivos programados con antelación a su ejecución, a la participación de la comunidad educativa y los elementos externos. Esto ofrecerá una visión global del proyecto no solo por el resultado conseguido o producto logrado, sino además por las carencias apreciadas (Gento, 1998).

Integrar saberes, desarrolla el conocimiento científico y la praxis en forma indiscutible, siempre que esta integración sea coherente y secuencial, lo que obliga a que la estructura curricular facilite dicha integración, por lo que es necesario hacer un análisis profundo de la malla curricular vigente en las universidades o escuelas politécnicas en la que se desee aplicar la integración, para del resultado del estudio, se pueda diagnosticar el éxito o fracaso, que sería la 
aplicación del proyecto integrador de saberes en cada nivel o semestre de la misma, lo que en forma directa o indirecta involucra a la institución y a los organismos de control de la educación de superior de Ecuador, en una corresponsabilidad de mejorar la estructura académica de todo el sistema en búsqueda de la calidad, excelencia y resultados de aprendizajes que generen egresados con perfiles de egreso acordes a las necesidades reales del mercado laboral, y luego profesionales con competencias útiles y atractivas para ese contexto

Desde el punto de vista empírico, la aplicación de todo este proceso de integración de saberes, genera una serie de beneficios y vivencias de alta relevancia en la construcción del conocimiento del futuro profesional, e incentivan metódica, sistemática y secuencialmente al mismo a generar aportes prácticos que solucionen problemas de la profesión, objetivo fundamental en la formación y el desarrollo de las habilidades necesarias, que le permitan ser competitivo en el contexto laboral real, e incluso podríamos decir emprendedor puesto que es una temática abordada en las propuestas.

Por lo expuesto se podría considerar al proyecto integrador como una técnica o instrumento es eficiente para la gestión formativa y participativa, pues promueve la investigación - acción, puesto que entrelaza un enfoque experimental con programas de acción, que responden a problemas, en este caso en un contexto real, generando así, los cambios requeridos y necesarios para mejorar o solucionar los mismos, es decir, una técnica que fusiona la formación teórica con lo práctico, y que se evidencia efectivamente en los resultados de aprendizajes, de acuerdo al nivel o semestre en que se aplique, corroborando así el grado de complejidad de la temática a resolver, lo que ratifica la construcción del conocimiento científico.

En el proyecto integrador de saberes el investigar parte de estudios previos o antecedentes de la misma y soporta teóricamente la propuesta, para dar el rigor científico a la misma, teorías que además de establecerse como una guía desde varios puntos de vista, en la elaboración de dicha investigación, permitirán estructurar una presuposición o hipótesis con base a teorías sustantivas aprobadas y probadas por la comunidad científica correspondiente, cuyos estudios actuales serán un aporte en la construcción de una propuesta eficiente considerando los procesos y eficacia teniendo en cuenta los resultados.

En cuanto a la elaboración de los instrumentos de recopilación de datos en el estudio de campo, será necesario que este concuerde con el enfoque y los resultados que deseamos obtener en la investigación o estudio, dado que los objetivos de la misma ya se plantearon previamente, y no olvidemos que debemos comprobar la hipótesis antes de hacer una propuesta, ya que de ser una hipótesis nula, tendríamos que replantear nuevas soluciones al problema, de allí la importancia de aplicar la metodología acorde en el desarrollo del proyecto integrador de saberes.

\section{Recomendaciones}

En base a lo expuesto se considera, que se debe aplicar; tanto la estructura y las estrategias metodológicas expresadas en el presente artículo en la planificación y elaboración de un Proyecto integrador de Saberes, ya que, de este modo el mismo se convierta en la evidencia del resultado del aprendizaje en cada nivel de formación, y en conjunto aporte al perfil de egreso. 
Sin embargo, es importante destacar que el proyecto integrador de saberes, además de integrar conocimientos en forma secuencial, nos obliga a que se involucre a la comunidad educativa en un proceso de indagación como manifiestan varios autores, idea que proviene de una insatisfacción, carencia o deficiencia en la gestión del estado actual de cosas, por lo que se debería inicialmente considerar el origen del problema que se desea estudiar o investigar y sus causas, es decir, un proceso analítico y lógico para descubrir el núcleo o falencia del mismo, y como consecuencia llegar a plantear las acciones a seguir para resolverlo, lo que nos conlleva a formular la pregunta o preguntas científicas, que deberán ser respondidas mediante la estructuración de los objetivos o metas de la investigación, tanto el o los objetivos generales como los específicos.

En todo proyecto de investigación, los aspectos metodológicos deberán ser acordes, conforme al contexto de aplicación, una tarea titánica y de responsabilidad compartida entre docente y discente, pero decisiva al momento de ejecutar el estudio de campo, desde el enfoque que se le dé al estudio o investigación, la tipología, el método, técnica e instrumento o instrumentos a aplicar en el mismo, por esto se podría deducir que la metodología de investigación seria el eje transversal en el desarrollo y estructura de los proyectos de integración de saberes.

Se debe regular su validación con normas y criterios que acrediten la gestión universitaria desarrollada con el proyecto, en el contexto y recursos tanto internos como externos, esta evaluación no estrictamente como un control, sino con el fin de mejorar su ejecución y afianzar niveles adecuados de calidad educativa (Ver figura A2). Se espera que esta obra sirva de sustento para otras investigaciones que permitan la evolución del tema, ya que resulta conveniente propiciar el brote de otros paradigmas que aporten a analizar, ampliar y mejorar los aspectos del Proyecto Integrador de Saberes.

\section{Bibliografía}

Arías, F. G. (2012). El proyecto de investigación (Sexta ed.). Caracas, Venezuela: Episteme.

Asamblea Nacional Constituyente. (2009). Ley Orgánica Reformatoria de la Disposición Transitoria Primera del Mandato Contituyente No. 14. Quito. Recuperado el 31 de Enero de 2017, de http://docs.ecuador.justia.com/nacionales/leyes/ley-organica-reformatoria-almandato-constituyente-n-14.pdf

Comisión Académica - Facultad de Ciencias Administrativas. (2017). Instructivo para el desarrollo de los Proyectos Integradores de Saberes a través de la catedra integradora de la Facultad de Ciencias Administrativas. Guayaquil: Universidad de Guayaquil.

Consejo de Educación Superior. (2013). Plan de Excelencia Universitaria. Quito: Lexis.

Consejo de Educación Superior. (2013). Reglamento de Régimen Académico. Quito : Lexis.

Consejo de Educación Superior. (12 de Noviembre de 2013). www.ces.gob.ec. Obtenido de www.ces.gob.ec: 
http://www.ces.gob.ec/doc/Reglamentos_Expedidos_CES/codificacin\%20del\%20reglam ento\%20de\%20rgimen\%20acadmico.pdf

Consejo de Evaluación, Acrediación y Aseguramiento de la Calidad de la Educación Superior, CEAACES. (2014). Reglamento de Evaluación, acreditación y categorización de carreras de las instituciones de educación superior. Quito: Lexis.

Consejo Nacional de Evaluación y Acreditación de la Educación Superior del Ecuador, CONEA. (2009). Mandato Constituyente No. 14. Quito: Lexis.

Coordinación de formación Facultad de Ciencias Admiistrativas - Universidad de Guayaquil. (2016). Instructivo para el desarrollo de proyectos integradores a través de la catedra integradora. Guayaquil: UG.

Cruz Rizo, L., \& Matos Hernández, E. C. (2017). Culminación de estudios: Una propuesta integradora en la Educación Superior. Revista científica Ciencia y Tecnología, 1-48. Obtenido de http://www.uteg.edu.ec/revista/index.php/cienciaytecnologia/article/view/85/77

Galeana, L. (Enero de 2017). ceupromed.ucol.mx. Recuperado el 31 de Enero de 2017, de ceupromed.ucol.mx: http://ceupromed.ucol.mx/revista/PdfArt/1/27.pdf

Gento, P. S. (1998). http://revistas.uned.es. Recuperado el 26 de Junio de 2017, de http://revistas.uned.es: file:///D:/_Usuarios/ERIKA/Downloads/399-1319-1-PB.pdf

Granados, E. L. (20 de Abril de 2015). CES.gob.ec. Obtenido de CES.gb.ec: http://www.ces.gob.ec/doc/Taller-difusion/SubidoAbril-2015/curriculo_essistemico\%20-\%20e\%20larrea.pdf

Larrea, E., \& Montalván, M. (2016). Modelo educativo ecológico de la Universidad de Guayaquil. Universidad de Guayaquil, Formación Académica. Guayaquil: UG.

Organización de las Naciones Unidas para la Agricultura y la Alimentación, FAO. (2011). fao.org. Recuperado el 31 de Enero de 2017, de fao.org: http://www.fao.org/docrep/006/t3725S/t3725s03.htm

Organización de las Naciones Unidas para la Educación, la Ciencia y la Cultura, UNESCO. (Mayo de 2011). www.ui.unesco.org. Obtenido de www.ui.unesco.org: http://www.uis.unesco.org/Education/Documents/isced-2011-sp.pdf

Piaget, J. (s.f.). $\quad w w w . u b . e d u$ Obtenido de www.ub.edu: http://www.ub.edu/dppsed/fvillar/principal/pdf/proyecto/cap_05_piaget.pdf

Presidencia de la República. (2010). Ley Orgánica de Educación Superior. Quito: Lexis. Obtenido de http://www.ups.edu.ec/documents/10184/19367/Ley+Org\%C3\%A1nica+de+Educaci\%C 3\%B3n+Superior/b691001e-b2fb-47b6-8f54-6e32331a2a5e 
Queipo, E. A. (Octubre de 2016). www.journaluidegye.com. Obtenido de www.journaluidegye.com:

http://www.journaluidegye.com/magazine/index.php/innova/article/view/108/76

Sampieri, R. H., Collado, C. F., \& Lucio, P. B. (2014). Metodología de la investigación (Sexta ed.). México D. F., México: Mc Graw Hill / International Editores S. A. de C. V.

Secretaría de Educación Superior, Ciencia, Tecnología e Innovación, SENECYT. (2015). www.snna.gob.ec. Obtenido de www.snna.gob.ec: http://www.snna.gob.ec/wpcontent/themes/institucion/comunicamos_noticias83.php

SENPLADES, Secretaría Nacional de Planificación y Desarrollo. (2013). Plan Nacional del Buen Vivir 2013 -2017 (Primera ed., Vol. 1). Quito, Pichincha, Ecuador: Senplades. Obtenido de www.buenvivir.gob.ec

\section{Apéndice}

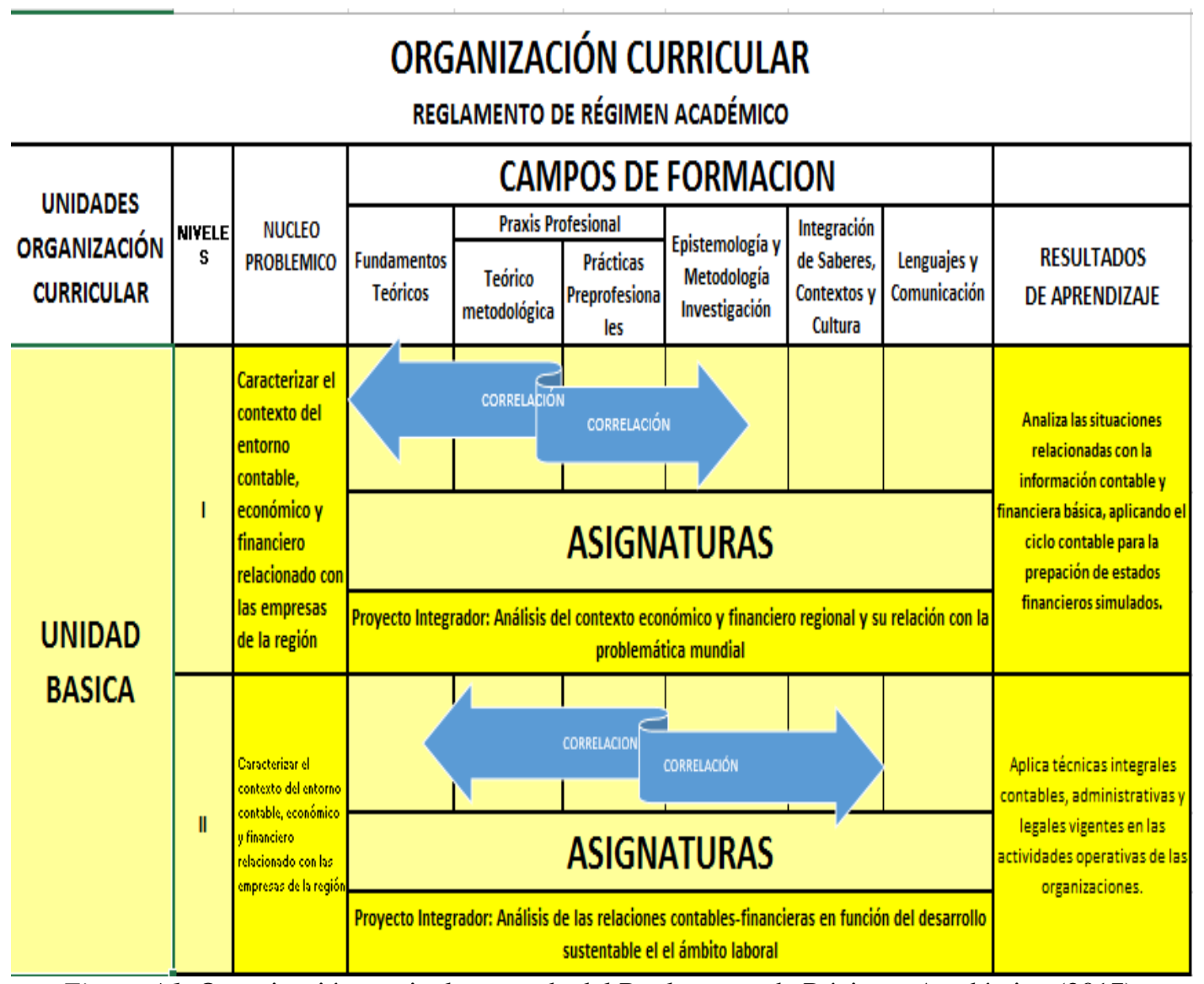

Figura A 1: Organización curricular tomada del Reglamento de Régimen Académico (2017). 


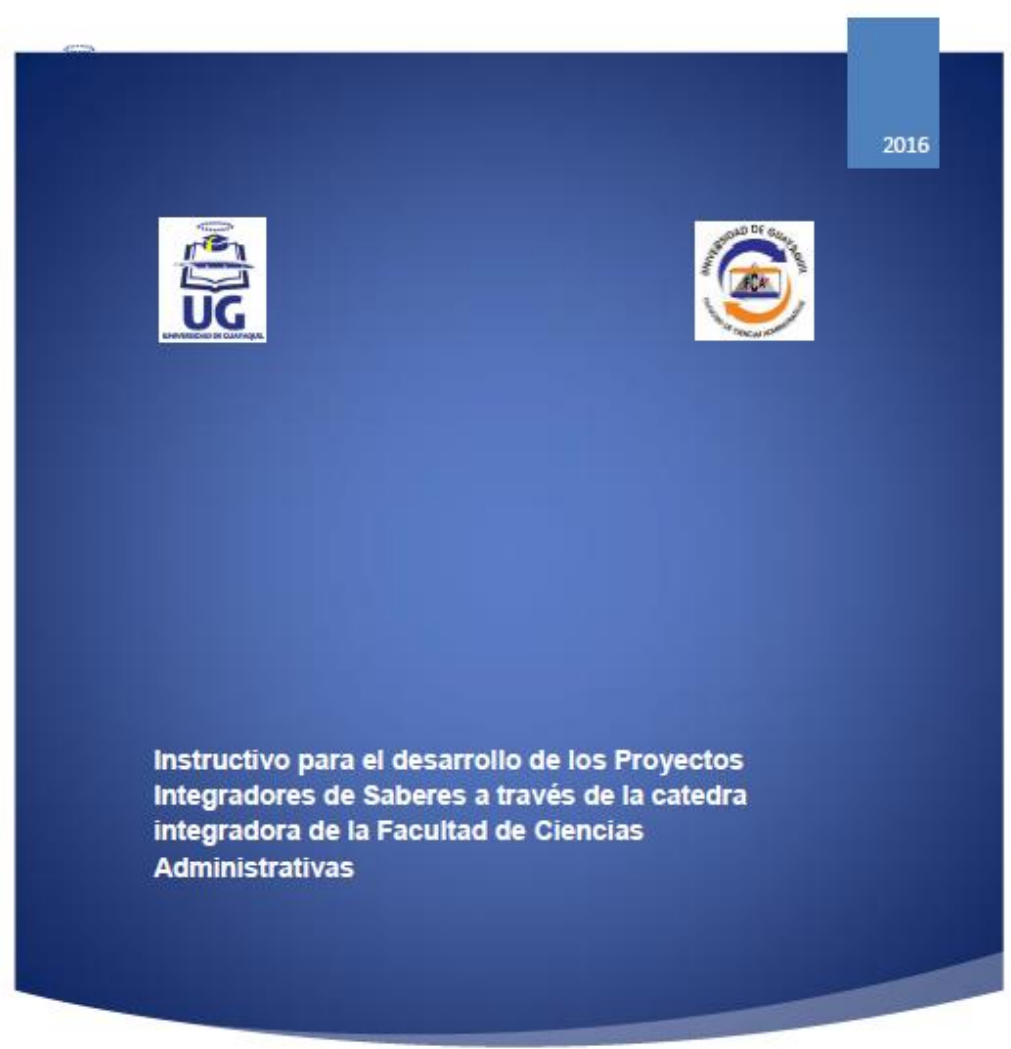

Figura A2: Portada de Guía del Proyecto Integrador de Saberes de la FCA de la Universidad de Guayaquil, donde se incluye la reglamentación para los estudiantes, docentes, gestores y autoridades en la elaboración de los PIS acordes a los niveles de enseñanza. También determina la catedra integradora para cada carrera por nivel y sus funciones, además contiene rubrica para la evaluación de las propuestas. Tomado de (Comisión Académica - Facultad de Ciencias Administrativas, 2017) 